\title{
A Numerical Study on the Extreme Intensification of Hurricane Patricia (2015)
}

\author{
K. RYDER FOX \\ New Mexico Institute of Mining and Technology, Socorro, New Mexico, and SOARS Program, \\ University Corporation for Atmospheric Research, Boulder, Colorado \\ FALKO JUDT \\ National Center for Atmospheric Research, Boulder, Colorado
}

(Manuscript received 17 July 2017, in final form 18 May 2018)

\begin{abstract}
In October 2015 Hurricane Patricia stormed through the eastern Pacific, taking its place as the strongest hurricane in recorded history when its intensity reached a record breaking $185 \mathrm{kt}\left(1 \mathrm{kt}=0.51 \mathrm{~m} \mathrm{~s}^{-1}\right)$. Operational models and the National Hurricane Center's official forecast failed to predict Patricia's unprecedented intensification, provoking questions as to whether such an extreme event can actually be forecast. This study reports on the successful simulation of Patricia using a state-of-the-art high-resolution numerical weather prediction model. It was found that high model resolution $(\Delta x \leq 1 \mathrm{~km})$, vortex initialization, and the parameterization of dissipative heating were key factors in realistically simulating Patricia's intensity evolution. The simulation was used to investigate Patricia's environment in terms of sea surface temperature, vertical wind shear, and humidity, under the premise that a simulation able to capture Patricia's peak intensity would also accurately represent Patricia's environment. Compared with a climatology derived from the Statistical Hurricane Intensity Prediction Scheme dataset, sea surface temperature ranked in the 99th percentile and environmental vertical wind shear in the 83rd percentile (ordered from high to low). However, humidity ranked more moderately. Ensemble forecasts indicate that Patricia had relatively high predictability in comparison to other well-studied rapid intensification cases such as 2010's Hurricane Earl. The results from this study imply that high-resolution models are in principle able to predict the intensity of extreme hurricanes like Patricia.
\end{abstract}

\section{Introduction}

Hurricane Patricia of 2015 (Fig. 1) was a recordbreaking eastern Pacific tropical cyclone (TC; Rogers et al. 2017; Kimberlain et al. 2016). Patricia's intensification rate of $105 \mathrm{kt}(24 \mathrm{~h})^{-1}\left(1 \mathrm{kt}=0.51 \mathrm{~m} \mathrm{~s}^{-1}\right)$ shattered the previous eastern Pacific TC intensification record of $75 \mathrm{kt}(24 \mathrm{~h})^{-1}$ (Hurricane Linda, 1997). Upon reaching a peak intensity of $185 \mathrm{kt}$, Patricia became the strongest TC ever observed in the Western Hemisphere. Despite quickly weakening before landfall, Patricia caused \$325 million (U.S. dollars) in damage across central Mexico, affecting over 10000 homes and $405 \mathrm{~km}^{2}$ of farmland (Kimberlain et al. 2016).

Significant improvements to hurricane track predictions in recent decades have not equivalently corresponded to improved intensity predictions. Particularly, rapid intensification (RI) remains a serious challenge (Rappaport et al. 2009). As a case in point, the operational forecast models

\footnotetext{
Corresponding author: K. Ryder Fox, k.ryder.fox@gmail.com
}

and the official NHC forecast missed Patricia's $\mathrm{RI}^{1}$ and grossly underpredicted Patricia's actual intensity (Fig. 1b, gray lines vs black line with triangles).

RI events are usually associated with favorable environmental conditions. Kaplan and DeMaria (2003) found Atlantic basin storms that undergo RI were typically located in environments with high sea surface temperatures (SSTs), high relative humidity (RH), and low vertical wind shear. Kaplan et al. (2010) updated the Kaplan and DeMaria (2003) study, extended it to the eastern North Pacific basin, and developed an index that estimates the probability of RI over the succeeding $24 \mathrm{~h}$ based on large-scale predictors from the Statistical Hurricane Intensity Prediction Scheme (SHIPS). The large-scale environment has relatively high predictability, lending to the predictive skill of RI forecasting tools such as the Kaplan et al. (2010) index. Particularly, Kaplan et al. (2015) found RI predictability was relatively

\footnotetext{
${ }^{1}$ Eastern Pacific TCs are said to undergo RI when their rate of intensification is at least $35 \mathrm{kt}(24 \mathrm{~h})^{-1}$ (Kaplan et al. 2010). Patricia's rate of $105 \mathrm{kt}(24 \mathrm{~h})^{-1}$ was 3 times this value.
} 
Hurricane Patricia (2015) Track \& Intensity
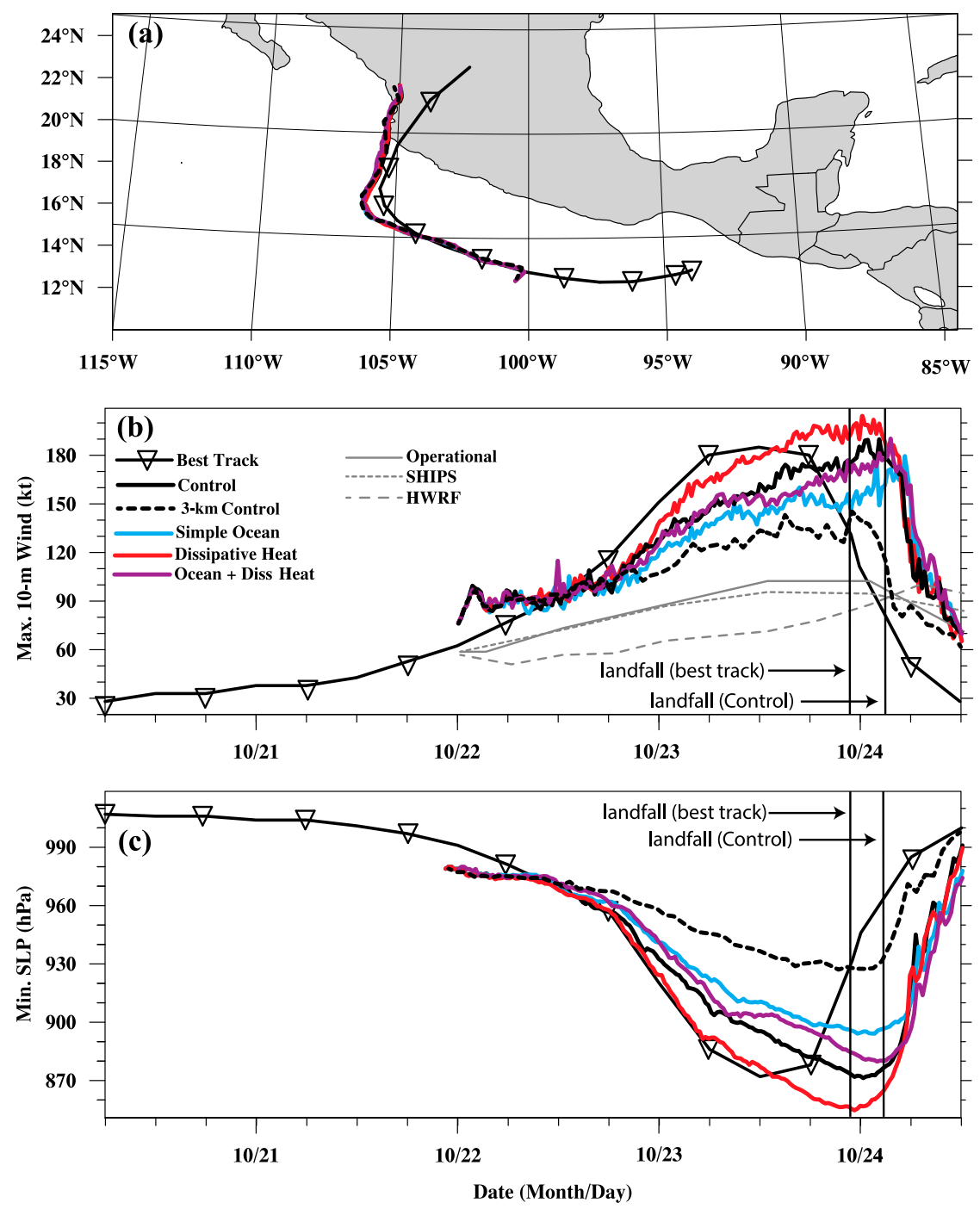

FIG. 1. Best-track data overlaid with track and intensity from the WRF simulations (black and colored lines) and the operational NHC, SHIPS, and HWRF forecasts from 0000 UTC 22 Oct 2015 (gray lines in Fig. 1b).

high for eastern North Pacific storms within favorable thermodynamic environments.

However, the environment is not the only factor contributing to RI. Recent research has indicated that RI is associated with smaller-scale processes (Rogers 2010; Guimond et al. 2010; Hazelton et al. 2017; Stevenson et al. 2014; Rogers et al. 2015). In particular, several studies link the significance of convective bursts to TC intensification (Steranka et al. 1986; Rodgers et al. 1998; Heymsfield et al. 2001; Chen and Zhang 2013). Smaller-scale processes generally pose a challenge for RI prediction because of their low predictability (Judt and Chen 2016). Some studies even suggest that it is virtually impossible to deterministically predict RI (e.g., Zhang and Tao 2013; Judt and Chen 2016).
Although TCs are atmospheric phenomena, TC intensity is regulated by the underlying ocean surface (e.g., Emanuel 1986), and some studies argue that air-sea processes need to be modeled explicitly in order to accurately predict TC intensity (e.g., Lee and Chen 2012). In some cases, oceanatmosphere interactions seemingly contribute to RI (e.g., Lin et al. 2009), highlighting the important role of the ocean.

Motivated by Patricia's extreme intensification and the failure of the operational models to predict the storm's intensity, the goals of this study are

1) to assess the ability of current numerical weather prediction models to capture Patricia's unprecedented intensity evolution, 
2) to investigate Patricia's environmental conditions, and 3) to explore Patricia's predictability.

In section 2, we provide information about the model and the experiment. Section 3 presents an evaluation of various model simulations, while section 4 assesses Patricia's environmental conditions. Section 5 investigates the overall predictability of this case. Finally, section 6 concludes the study with a summary.

\section{Methodology}

\section{a. Model configuration and experiment setup}

The foundation of this work is a set of high-resolution numerical model simulations produced with version 3.8 of the Advanced Research version of the Weather Research and Forecasting (WRF) Model (Skamarock et al. 2008). Following a standard approach in hurricane modeling (Davis et al. 2008), WRF was configured with one outer fixed domain and two inner vortex-following nests. Similar to Judt et al. (2016), the vertical dimensions of the WRF Model used for this study featured 44 levels with higher vertical resolution in the boundary layer and near the melting level. The model's outer domain had dimensions of $450 \times 350$ grid points with a grid spacing of $9 \mathrm{~km}$, covering an area of $4050 \mathrm{~km} \times 3150 \mathrm{~km}$ (Fig. 2, outermost box). The two inner, vortex-following domains (Fig. 2, inner black boxes) had dimensions of $300 \times 300$ grid points with grid spacings of 3 and $1 \mathrm{~km}$, respectively. The physics parameterizations used in this experiment are listed in Table 1. We chose parameterization schemes standard to convection-permitting WRF simulations and similar to those of Judt et al. (2016). In particular, the Tiedtke convection scheme is used only in the outermost domain. Additionally, the SSTs in the control simulation came from the Global Forecast System (GFS) reanalysis and were fixed throughout the run.

The WRF Model's default version (with which the control run was produced) does not feature ocean coupling or include the effect of dissipative heating. ${ }^{2}$ However, studies show that both factors affect hurricane intensity (e.g., Lee and Chen 2012; Bister and Emanuel 1998). We therefore produced three additional experimental simulations to estimate the error induced by neglecting these processes:

1) The WRF single-column mixed layer ocean model was turned on.

2) The dissipative heating option was turned on. ${ }^{3}$

3) Both options were turned on simultaneously.

\footnotetext{
${ }^{2}$ Heating is by frictional transfer of kinetic energy at the molecular level.

${ }^{3}$ Results are represented in the WRF code according to Eq. (6) in Zhang and Altshuler (1999).
}

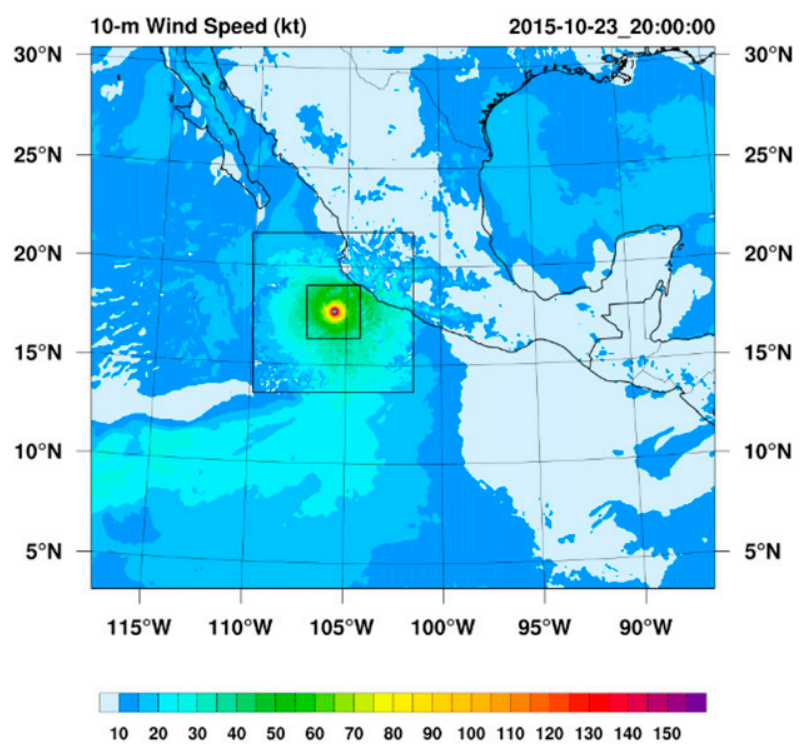

FIG. 2. The 10-m wind speed from the WRF control simulation around the time Patricia reached peak intensity in the simulation (0000 UTC 24 Oct 2015). The inner vortex-following domains with 3 - and 1-km grid spacing are indicated by black squares.

To address the question of whether grid spacing of $1 \mathrm{~km}$ is necessary to successfully capture Patricia's peak intensity, we produced an additional 3-km run. The lowerresolution run was similar to the control run, except that the innermost nest was not activated. Finally, to investigate Patricia's predictability, we generated two ensemble forecasts. The first ensemble, which had five members, was generated by stochastically perturbing the temperature field of the initial conditions with random noise (mean $=0$; standard deviation $=0.02 \mathrm{~K}$ ). This method is commonly used to study intrinsic predictability (e.g., Zhang et al. 2003; Selz and Craig 2015). The second ensemble, which had 15 members, was generated using the stochastic energy backscatter scheme (SKEBS; Shutts 2005; Berner et al. 2009, 2011), with the same model domains and grid spacing mentioned earlier. In contrast to the first ensemble where only the initial conditions were perturbed with miniscule random noise, SKEBS perturbs the model fields with random, small-amplitude perturbations to the horizontal wind and potential temperature tendency equations at each time step. Judt et al. (2016) and Judt and Chen (2016) employed SKEBS to examine the predictability of Hurricane Earl (2010). Those Earl ensembles will serve as a comparison of the WRF's performance with regard to predicting Patricia.

\section{b. Vortex relocation}

The control simulation and ensembles were initialized at 0000 UTC 22 October 2015 with $0.25^{\circ}$-resolution reanalysis fields from the GFS. The initialization time was chosen because it was reasonably close to the onset of Hurricane Patricia's RI while still early enough that the 
TABLE 1. List of physics parameterizations.

\begin{tabular}{|c|c|}
\hline Parameterization & Reference \\
\hline Tiedke convective scheme & $\begin{array}{l}\text { Tiedtke (1989), } \\
\text { Zhang et al. (2011) }\end{array}$ \\
\hline $\begin{array}{l}\text { Rapid radiation transfer } \\
\text { longwave and shortwave } \\
\text { radiation schemes }\end{array}$ & Iacono et al. (2008) \\
\hline $\begin{array}{l}\text { Yonsei University (YSU) } \\
\text { planetary boundary layer } \\
\text { scheme }\end{array}$ & Hong et al. (2006) \\
\hline $\begin{array}{l}\text { Revised MM5 surface-layer scheme; } \\
\text { surface bulk drag and enthalpy } \\
\text { coefficients modified to be more } \\
\text { in line with recent research results } \\
\text { of those for hurricanes }\end{array}$ & $\begin{array}{l}\text { Fairall et al. (2003), } \\
\text { Donelan et al. (2004) }\end{array}$ \\
\hline $\begin{array}{l}\text { WRF single-moment 6-class } \\
\text { (WSM6) microphysics scheme }\end{array}$ & Hong and Lim (2006) \\
\hline
\end{tabular}

model could produce the intensification on its own. Put differently, initializing the model earlier would miss RI, while initializing closer to RI onset would artificially "force" the phenomenon due to RI being ingrained in the initial conditions. During the experimental stage of this study, we noticed that a simulation initialized solely with GFS fields could not accurately capture the mesoscale structure of Patricia's vortex, which resulted in an incorrect intensity evolution. In response, we employed the vortex relocation method of Liu et al. (2000). For this purpose, a precursory WRF simulation was initialized with GFS fields from 1200 UTC 21 October 2015. This precursory simulation was used to spin up a TC with a more realistic mesoscale structure. The 12th-hour $(0000$ UTC 22 October) vortex of the precursory simulation was then used to replace the original GFS vortex within the 0000 UTC 22 October 2015 (control) WRF initial condition file. We terminated all runs after Patricia's landfall, at 1200 UTC 24 October 2015 and, with the exception of the precursory run, all simulations lasted a total of $60 \mathrm{~h}$.

\section{c. Aircraft data}

We facilitated a model evaluation beyond the standard "best track" metrics with hurricane hunter aircraft observations retrieved from NOAA's Hurricane Research Division website (http://www.aoml.noaa.gov/hrd/ Storm_pages/patricia2015/). Specifically, we used in situ observations of flight-level wind speed and sea level pressure (SLP) from a NOAA P-3 mission and a U.S. Air Force (USAF) C-130 mission, chosen for their proximity to Patricia's peak intensity (the aircraft were on station around 0600 and 1800 UTC 23 October 2015, respectively).

\section{d. SHIPS climatology}

To compare the environmental conditions during Patricia's RI with the environments of other eastern Pacific TCs, we juxtaposed environmental parameters from the WRF control simulation with climatological data obtained from the SHIPS eastern North Pacific dataset (Kaplan et al. 2010; http:// rammb.cira.colostate.edu/research/tropical_cyclones/ships/ developmental_data.asp). We chose all TCs within the dataset for this study in order to make a robust comparison of Patricia's environment at the time of its intensification. The environmental parameters that we assessed, SST, vertical wind shear, and low- and midlevel RH, corresponded to the following SHIPS predictors: RSST, SHRD, RHLO, and RHMD. In particular, we used the WRF Model output to calculate these environmental parameters using the same method as SHIPS. Specifically for SST, the center point of the innermost domain was recorded, while shear and $\mathrm{RH}$ were calculated within a 200-800-km annulus around Patricia's center (DeMaria et al. 2005). Time averaging for our analyses was centered around the 24-h RI periods associated with the observed (0600 UTC 22 October-0600 UTC 23 October) and modeled (0000 UTC 23 October-0000 UTC 24 October) storms, respectively.

\section{Model evaluation}

Aside from a minor westward bias, the control and experimental simulations reproduced Patricia's track, including the rightward turn toward the Mexican coast (Fig. 1a). All simulations captured Patricia's intensification including the RI period; yet, there are systematic differences between the runs (Figs. 1b,c). The control run (black) reached a maximum wind speed of $190 \mathrm{kt}$ and a minimum SLP of $871 \mathrm{hPa}$ (Figs. 1b,c), both remarkably close to Patricia's actual peak intensity $(185 \mathrm{kt}, 872 \mathrm{hPa})$. However, the simulated storm's peak intensity occurred $\sim 12 \mathrm{~h}$ later than observed, and all but the simulation including dissipative heating failed to fully reproduce Patricia's intensification rate. Furthermore, given that the model did not capture the observed secondary eyewall formation (a secondary eyewall had formed by 2023 UTC 23 October; Kimberlain et al. 2016), none of the simulations captured the overwater weakening.

Not surprisingly, the simulation that includes ocean coupling (Fig. 1, blue) featured an overall weaker hurricane due to the negative SST feedback (Emanuel 1988). In contrast, accounting for dissipative heating (Fig. 1, red) led to a stronger storm. When both ocean coupling and dissipative heating were activated (Fig. 1, purple), the two effects nearly canceled each other out, and the storm's intensity evolution followed that of the control simulation. This suggests that the control run's success in reproducing Patricia's peak intensity owed itself, in part, to "error compensation," meaning that neglecting ocean coupling and dissipative heating results in intensity biases in opposite directions that cancel each other out. 
a) Wind Speed $(700 \mathrm{hPa})$

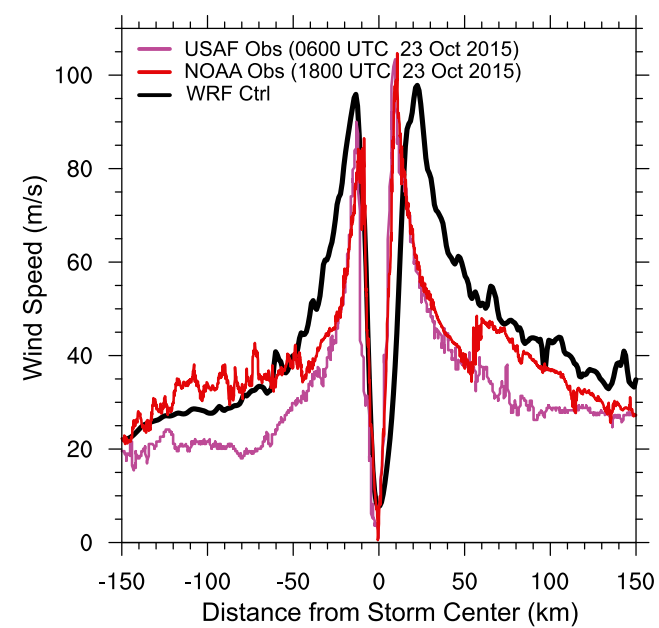

b) Sea Level Pressure

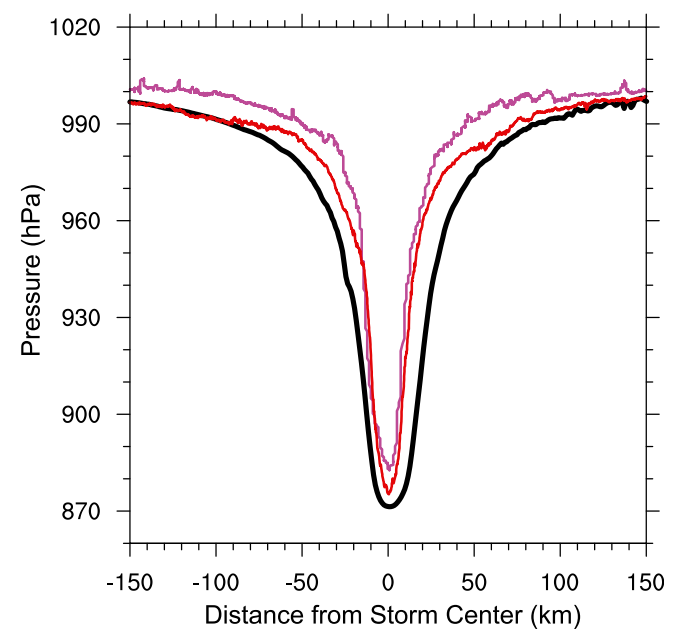

FIG. 3. Cross sections through Patricia's (a) 700-hPa wind and (b) SLP fields. The black line is the WRF control simulation, and red and magenta lines indicate aircraft observations. The observations were taken $6 \mathrm{~h}$ before (0600 UTC 23 Oct 2015) and after (1800 UTC 23 Oct 2015) Patricia was estimated to have reached peak intensity, whereas the model data correspond to the time when the simulated Patricia reached peak intensity (0000 UTC 24 Oct 2015).

A grid spacing of at least $1 \mathrm{~km}$ seems necessary to capture Patricia's peak intensity, as noted by the simulation produced with the $3-\mathrm{km}$ domain only (Fig. 2). The peak intensity of the storm in the $3-\mathrm{km}$ simulation was at least $30 \mathrm{kt}$ higher than the operational model predictions. However, it only reached a peak wind speed of around $130 \mathrm{kt}$ and a minimum SLP of around $930 \mathrm{hPa}$, not even close to the observed values or the intensity of the 1-km control run (Figs. 1b,c). While future work will investigate this further, it is likely that $1-\mathrm{km}$ grid spacing is needed to resolve the TC inner-core processes necessary to produce an accurate intensity forecast.

Compared to observations from two hurricane hunter aircraft on station approximately $6 \mathrm{~h}$ before and $6 \mathrm{~h}$ after (e.g., USAF, 0600 UTC 23 October; NOAA, 1800 UTC 23 October) Patricia's estimated peak intensity, we found that the control simulation captured Patricia's general peak intensity structure when comparing the wind and minimum SLP fields with aircraft observations. However, the simulation could not replicate the storm's exceedingly tight inner core or its exceptional gradients (Fig. 3). The simulated wind and pressure fields were broader than the observations, and somewhat smoother, while the eye was larger than observed. Given Skamarock's (2004) finding that WRF's effective resolution is on the order of $7 \Delta x$, the failure to precisely capture Patricia's inner-core gradients is not surprising. These biases suggest that even higher resolution is necessary to simulate the extreme gradients present in very small and intense hurricanes like Patricia.

\section{Environmental conditions}

\section{a. Environmental conditions during RI}

Kaplan and DeMaria (2003) showed that the majority of RI events occurred with SSTs $>27^{\circ} \mathrm{C}$, environmental shear $<3.8 \mathrm{~m} \mathrm{~s}^{-1}$, and low-level RH $>70 \%$. To better understand the role that the environment played in Patricia's intensification, we examined the corresponding quantities based on the WRF control simulation and valid at 0000 UTC 23 October. The analysis showed that Patricia was located in a generally favorable environment for RI. For example, the SSTs along Patricia's track were $>30^{\circ} \mathrm{C}$, with isolated areas exceeding $31^{\circ} \mathrm{C}$ (Fig. 4a).

To analyze vertical wind shear, we subtracted the $u$ and $v$ wind components at $850 \mathrm{hPa}$ from those at $200 \mathrm{hPa}$ and compared the magnitude of the vector wind difference. The resulting field in Fig. 4b shows that Patricia was located in an area of low shear. The broad anticyclonic turning of the shear vectors inside the domain signifies an upper-level anticyclone above the hurricane, usually a sign of a favorable kinematic environment. Higher shear magnitudes in excess of $40 \mathrm{~m} \mathrm{~s}^{-1}$ to the north of the cyclone seem to be associated with a jet that interacted with Patricia after landfall, contributing to its rapid weakening (not shown).

Next, we analyzed the low- and midlevel RH. For lowlevel RH, we averaged the 850- and 700-hPa-layer RH fields, and for the midlevel $\mathrm{RH}$, we averaged the 700and 500-hPa-layer RH fields. As seen in Fig. 4c, lowlevel RH in the vortex and its vicinity was at least $80 \%$, 
a) $\operatorname{SST}\left({ }^{\circ} \mathrm{C}\right)$

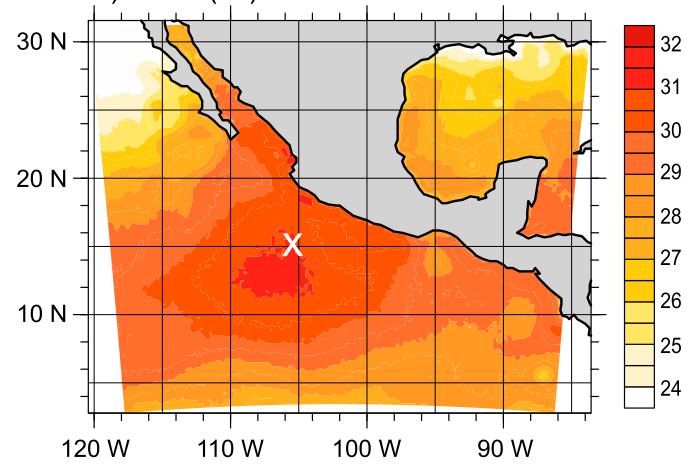

c) Low-Level RH (\%)

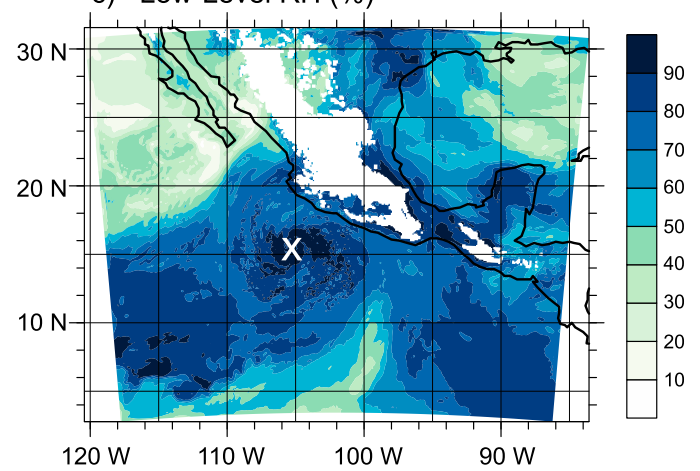

b) Local shear $(\mathrm{m} / \mathrm{s})$

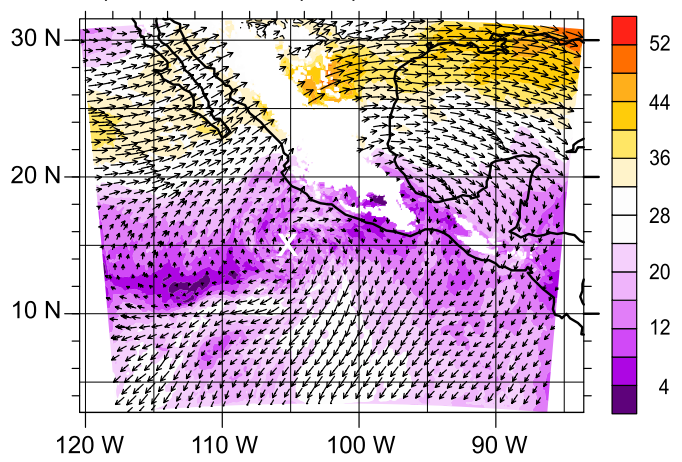

d) Mid-Level RH (\%)

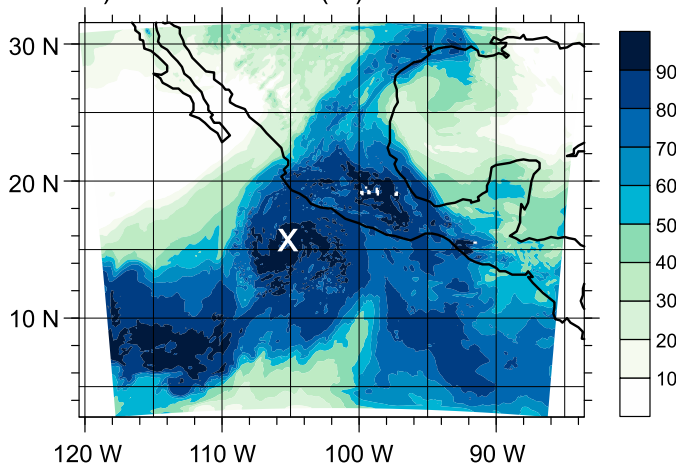

FIG. 4. WRF Model fields of (a) SST, (b) vertical wind shear, (c) low-level RH, and (d) midlevel RH at the time of Patricia's RI onset in the simulation (0000 UTC 23 Oct 2015). The white crosses indicate the center location of Patricia.

while it was above $90 \%$ in the core (Fig. 4c). The environment to the west and northwest of Patricia's vortex was driest, with $\mathrm{RH}<50 \%$. Similarly, midlevel RH values (Fig. 4d) were generally $>80 \%$, with $>90 \%$ in the core region but especially dry air $(<20 \%$ moisture $)$ north and northwest of the core. The transition to drier midlevel air was quite drastic on the western side of the hurricane. However, low shear prevented dry air from being advected into the inner core.

\section{b. Comparison with climatology}

In section 4a we highlighted the high SSTs, low shear, and moderate RH that characterized Patricia's environment. Here, we will rank those characteristics with respect to the SHIPS climatology in order to place Patricia's environment in perspective with respect to the environments of historical TCs. Kaplan et al. (2010) showed that the 94th percentile of eastern North Pacific overwater intensity change values had a mean environmental shear value of $3.8 \mathrm{~m} \mathrm{~s}^{-1}$ and a mean low-level RH value of $78.6 \%$. As no mean value for SST in RI cases was calculated for the eastern North Pacific basin, we used the Kaplan and DeMaria (2003) mean value $\left(28.4^{\circ} \mathrm{C}\right)$ calculated for the northern Atlantic basin as a comparison for Patricia's mean value.
Figure 5 shows the percentiles of the four environmental parameters previously discussed: SST, vertical wind shear, and low- and midlevel RH. The point values (SST) and spatial averages (shear, $\mathrm{RH}$ ) were calculated hourly during the $24 \mathrm{~h}$ leading up to Patricia's peak intensity and averaged over this time. The bins these values fall into are highlighted with black arrows in Fig. 5. At a value of $30.8^{\circ} \mathrm{C}$, Hurricane Patricia's averaged SST was in the 99th percentile of all eastern Pacific hurricanes (Fig. 5a), a high value compared to the RI mean of $28.4^{\circ} \mathrm{C}$. We furthermore compared the SST from the control simulation to the SHIPS predictor data (each averaged over their respective 24-h RI periods), finding that the values are reasonably close to each other (Table 2).

Environmental shear ranked as the second-most extreme of the WRF control simulation parameters. The shear value actually dropped to near zero for a brief period during RI, and when averaged over the $24 \mathrm{~h}$ leading up to Patricia's peak intensity, the value of $3.3 \mathrm{~m} \mathrm{~s}^{-1}$ was found to be $14 \%$ lower than the eastern Pacific RI mean. As such, the time average represents the 83 rd percentile (with $83 \%$ of all records exhibiting shear $>4 \mathrm{~m} \mathrm{~s}^{-1}$; Fig. 5b). We attributed the rise in shear value during the simulated Patricia's RI to a large area of higher shear found south and west of Patricia's 

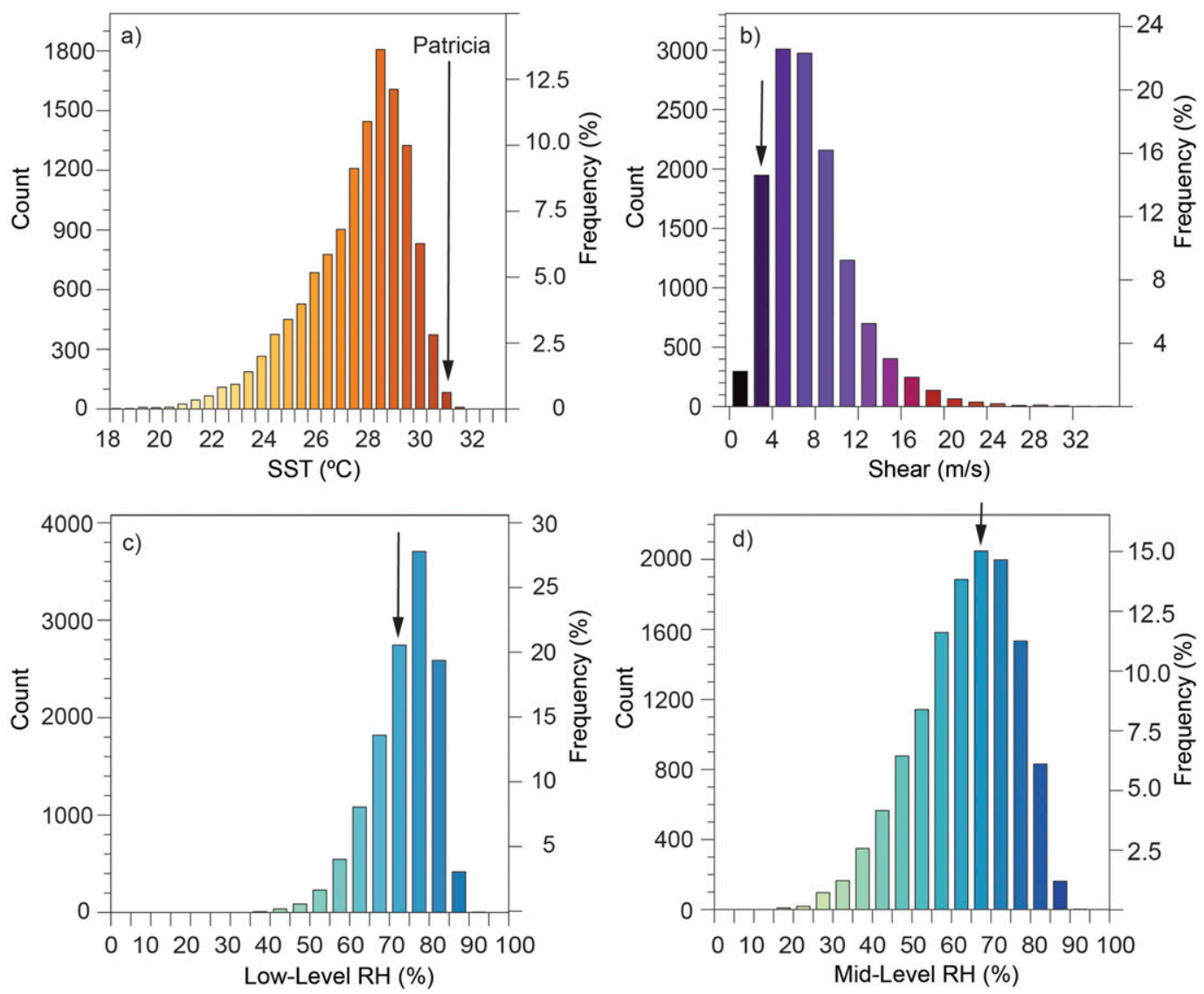

FIG. 5. Frequency distributions of (a) SST, (b) environmental vertical wind shear magnitude, (c) low-level RH, and (d) midlevel RH based on the SHIPS climatology (all northeastern Pacific TCs between 1982 and 2015). The arrows point to the bins Patricia's environmental conditions fall into (data from the WRF control simulation). Patricia's averages are calculated at the center point of the innermost domain (SST) and within a 200-800-km annulus (vertical wind shear and low- and midlevel RH).

vortex, which leads to the question of how representative this actual shear value is given that the high shear value was fairly far removed from Patricia's core. The limit on the radius of environmental air entrainment due to strong inertial stability in intense TCs has been previously shown (Riemer and Montgomery 2011). Comparing the control run to actual SHIPS values (Table 2), we found that the average shear value of $2.9 \mathrm{~m} \mathrm{~s}^{-1}$ in the SHIPS predictor file to be $12 \%$ lower.

We found more moderate rankings with respect to low- and midlevel RH. The low-level RH was $74.5 \%$, ranking least impressively, when compared to historical eastern Pacific TCs. The value fell into the 50th percentile (Fig. 5c) and slightly below the eastern Pacific RI mean of $78.6 \%$ moisture. The midlevel $\mathrm{RH}$, with a value of $68.9 \%$, ranked notably higher in the 66th percentile (Fig. 5d). Table 2 shows the comparisons between the data from the control simulation and the SHIPS predictor file, indicating only slight differences of no more than $5 \%$. Given the modest rankings of the $\mathrm{RH}$ values, which are mainly due to a dry environment relatively far from Patricia's core (Figs. 4c,d), it is possible that the relatively large averaging area of the static annulus led to an inadequate representation of Patricia's environment (especially in light of Patricia's small size).

\section{Predictability}

The ability of the WRF Model to successfully simulate Patricia suggests that high-resolution numerical weather

TABLE 2. Climatology comparison.

\begin{tabular}{lcc}
\hline \hline & Control & SHIPS predictors \\
\hline SST $\left({ }^{\circ} \mathrm{C}\right)$ & 30.8 & 30.4 \\
Vertical wind shear $\left(\mathrm{m} \mathrm{s}^{-1}\right)$ & 3.3 & 2.9 \\
Low-level RH $(\%)$ & 74.5 & 75 \\
Midlevel RH $(\%)$ & 68.9 & 72.6 \\
\hline
\end{tabular}



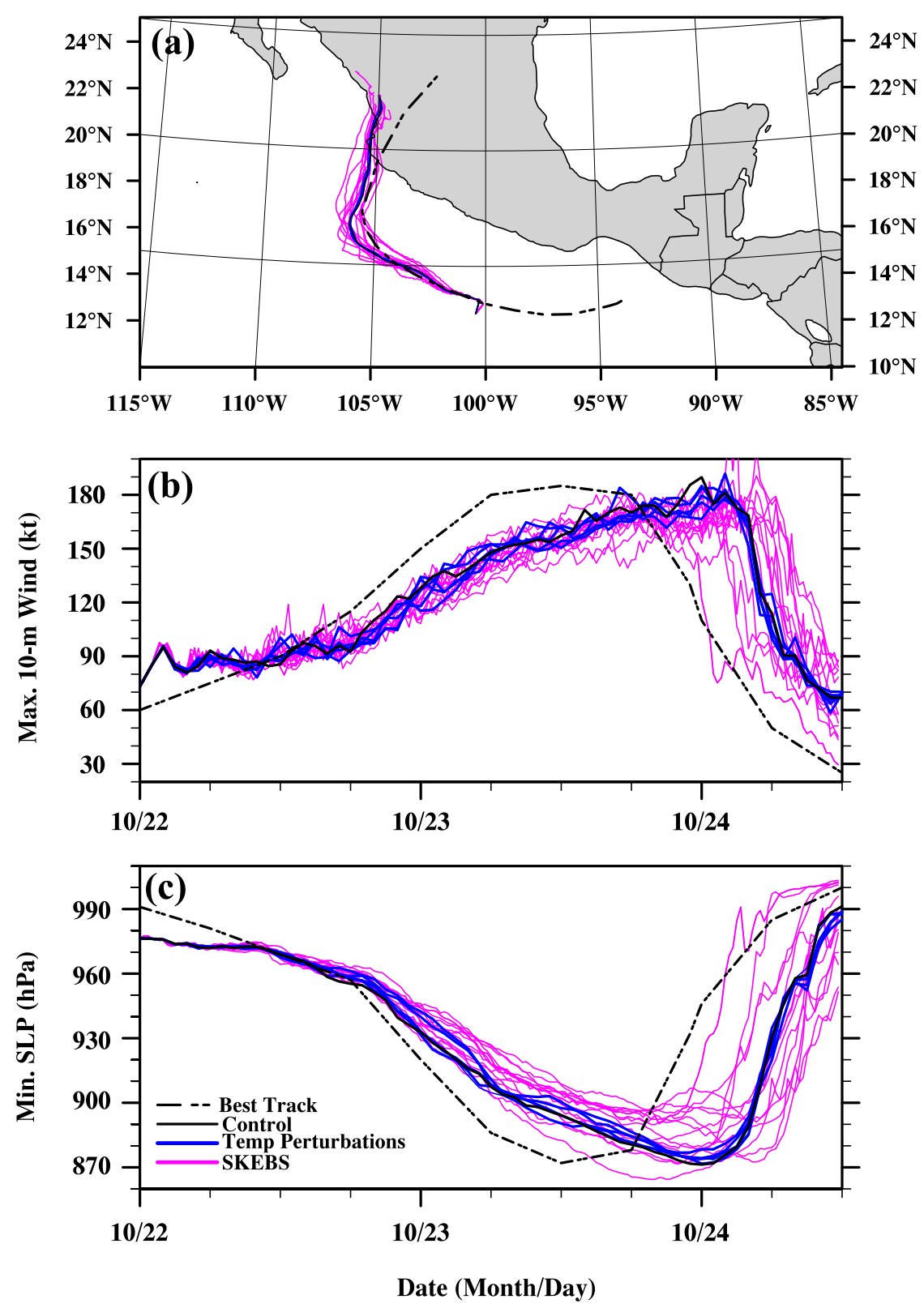

FIG. 6. Track and intensity of the WRF control simulation (black) overlaid with the 5-member ensemble (blue) and the 15-member SKEBS ensemble (magenta).

prediction models are, in principle, able to predict the intensity evolution of extreme TCs. The question remains whether this success was simply due to serendipity. One way to address the robustness of the results is to investigate the predictability of this case.

To examine Patricia's intrinsic predictability, track and intensity time series of the five-member ensemble that was generated with stochastic initial condition perturbations are shown in Fig. 6 (blue). Variability among the tracks is nearly nonexistent, supporting the findings of Judt et al. (2016), who showed that track uncertainty results from explicitly perturbing synoptic scales. Quite surprisingly, even the intensity time series show remarkably little spread. This finding differs from previous predictability studies, which emphasized the limited intrinsic predictability of TC formation and intensification (e.g., Zhang and Sippel 2009; Judt et al. 2016; Judt and Chen 2016; Munsell et al. 2017). Evidently, this particular case has higher intrinsic predictability compared to other TCs.

Patricia's predictability was further examined using a 15-member ensemble generated with the SKEBS scheme. The scheme was set up with parameters similar to the one 
that produced the "SKEBS-allScales" ensemble in Judt and Chen (2016; Fig. 6, magenta). Judt and Chen's (2016) case study of Hurricane Earl (2010), and their Figs. 1b and $1 \mathrm{c}$, in particular, are used here as a benchmark to compare with the SKEBS Patricia ensemble.

The Patricia SKEBS ensemble showed greater track and intensity variability than the ensemble with randomly perturbed initial conditions (Fig. 6). However, in comparison to the Hurricane Earl SKEBS ensemble, Patricia showed less intensity uncertainty. Of particular note was the ability of the Patricia SKEBS ensemble to capture Patricia's intensification with all ensemble members with a relatively small spread. The Earl ensemble members show a much wider distribution. In particular, the onset of $\mathrm{RI}$ is very uncertain in Earl, as indicated by the 3-day time window during which RI commences (Judt and Chen 2016, their Figs. 1b,c). In contrast, the RI onset uncertainty in the Patricia ensemble merely stretched over a few hours.

One reason for the higher predictability may be Patricia's environment. Judt and Chen (2016) demonstrated that a favorable environment could increase the predictability of $\mathrm{RI}$ in the sense that when the environment is predictable, so is the likelihood of RI. Along the same lines, Zhang and Tao (2013) make the case for the high intrinsic predictability of TC intensity for idealized cases in no-shear environments.

\section{Summary and conclusions}

Hurricane Patricia (2015) experienced an unprecedented period of rapid intensification (RI), propelling it into position as the strongest hurricane in recorded history. Operational weather prediction models failed to forecast both Patricia's rate of intensification and peak intensity. We simulated Patricia using the Weather Research and Forecasting (WRF) Model and demonstrated that WRF is capable of capturing the storm's intensity evolution. The simulations reproduced Patricia's peak intensity, albeit $12 \mathrm{~h}$ later than observed. The success of the simulations was due in part to initialization with a realistic vortex, in this case by the relocation of a vortex relocation, spun up in a precursory WRF simulation 1-km grid spacing, and accurately capturing the environmental conditions.

Analysis of the model output using the Statistical Hurricane Intensity Prediction Scheme (SHIPS) showed that Patricia's environment was generally favorable for intensification. In particular, high SSTs of $30.8^{\circ} \mathrm{C}$, which ranked in the 99th percentile, seem to have fueled the storm. Patricia's environmental shear of $3.3 \mathrm{~m} \mathrm{~s}^{-1}$ ranked in the lower 83rd percentile, indicating very low shear values during RI. The RH ranked most moderately, with the lowlevel RH average of $74.5 \%$ representing the 50th percentile, and the midlevel $\mathrm{RH}$ average of $68.9 \%$ falling into the 66th percentile. The moderate findings suggest that more than the environment might have contributed to Patricia's particularly extreme intensification and that the TC's internal-core processes may warrant further investigation. Also under consideration are the limitations of time averaging over the full 24-h RI period, as well as using a static annulus to evaluate the environments of TCs, like Patricia, with small inner cores.

In contrast to other well-studied RI events such as 2010's Hurricane Earl, the intensity evolution of this case has high predictability. This was demonstrated with ensembles featuring 1) random initial conditions perturbations and 2) continuous stochastic perturbations of the model fields during run time (SKEBS scheme). Since this study is limited to a single initialization time, future work will seek to generalize these results with other initial vortex representations for the Patricia case study as well as using similar RI cases to confirm whether the accurate intensity prediction of this case study is representative. Nonetheless, we demonstrated that there is hope for better hurricane intensity forecasts when high-resolution numerical models are initialized with realistic TC vortices and accurate states of the environment.

Acknowledgments. This work was performed under the auspices of the Significant Opportunities in Atmospheric Research and Science (SOARS) program, NSF Grant AGS-1120459, and the National Center for Atmospheric Research. We thank Chris Davis, David Ahijevych, Yangyang $\mathrm{Xu}$, and Mary Haley for their support of this work. We would like to acknowledge the use of computational resources (ark:/85065/d7wd3xhc) at the NCARWyoming Supercomputing Center provided by the National Science Foundation and the state of Wyoming, and supported by NCAR's Computational and Information Systems Library.

\section{REFERENCES}

Berner, J., G. J. Shutts, M. Leutbecher, and T.N. Palmer, 2009: A spectral stochastic kinetic energy backscatter scheme and its impact on flow-dependent predictability in the ECMWF Ensemble Prediction System. J. Atmos. Sci., 66, 603-626, https://doi.org/ 10.1175/2008JAS2677.1.

— S.-Y. Ha, J. P. Hacker, A. Fournier, and C. Snyder, 2011: Model uncertainty in a mesoscale ensemble prediction system: Stochastic versus multiphysics representations. Mon. Wea. Rev., 139, 1972-1995, https://doi.org/10.1175/2010MWR3595.1.

Bister, M., and K. A. Emanuel, 1998: Dissipative heating and hurricanes. Meteor. Atmos. Phys., 65, 233-240, https://doi.org/ 10.1007/BF01030791.

Chen, H., and D.-L. Zhang, 2013: On the rapid intensification of Hurricane Wilma (2005). Part II: Convective bursts and the upper-level warm core. J. Atmos. Sci., 70, 146-172, https://oi.org/ 10.1175/JAS-D-12-062.1.

Davis, C., and Coauthors, 2008: Prediction of landfalling hurricanes with the Advanced Hurricane WRF Model. Mon. Wea. Rev., 136, 1990-2005, https://doi.org/10.1175/2007MWR2085.1. 
DeMaria, M., M. Mainelli, L.K. Shay, J.A. Knaff, and J. Kaplan, 2005: Further improvements to the Statistical Hurricane Intensity Prediction Scheme (SHIPS). Wea. Forecasting, 20, 531-543, https://doi.org/10.1175/WAF862.1.

Donelan, M. A., B. K. Haus, N. Reul, W. J. Plane, M. Stiassnie, H. C. Grabber, O. B. Brown, and E. S. Saltman, 2004: On the limiting aerodynamics roughness on the ocean in very strong winds. Geophys. Res. Lett., 31, LI18306, https://doi.org/10.1029/ 2004 GL019460.

Emanuel, K. A., 1986: An air-sea interaction theory for tropical cyclones. Part I: Steady-state maintenance. J. Atmos. Sci., 43, 585-604, https://doi.org/10.1175/1520-0469(1986)043<0585: AASITF $>2.0 . \mathrm{CO} ; 2$.

- 1988: Toward a general theory of hurricanes. Amer. Sci., 76, 371-379.

Fairall, C. W., E. F. Bradley, J. E. Hare, A. A. Grachev, and J. B. Edson, 2003: Bulk parameterization of air-sea fluxes: Updates and verification for the COARE algorithm. J. Climate, 16, 571-591, https://doi.org/10.1175/1520-0442(2003)016<0571: BPOASF $>2.0 . \mathrm{CO} ; 2$.

Guimond, S. R., G. M. Heymsfield, and T. Joseph, 2010: Multiscale observations of Hurricane Dennis (2005): The effects of hot towers on rapid intensification. J. Atmos. Sci., 67, 633-654, https://doi.org/10.1175/2009JAS3119.1.

Hazelton, A. T., R. E. Hart, and R. F. Rogers, 2017: Analyzing simulated convective bursts in two Atlantic hurricanes. Part II: Intensity change due to bursts. Mon. Wea. Rev., 145, 30953117, https://doi.org/10.1175/MWR-D-16-0268.1.

Heymsfield, G. M., J. B. Halverson, J. Simpson, L. Tian, and T. P. Bui, 2001: ER-2 Doppler radar investigations of the eyewall of Hurricane Bonnie during the Convection and Moisture Experiment-3. J. Appl. Meteor., 40, 1310-1330, https://doi.org/ 10.1175/1520-0450(2001)040<1310:EDRIOT>2.0.CO;2.

Hong, S.-Y., and J. O. J. Lim, 2006: The WRF single-moment 6-class microphysics scheme (WSM6). J. Korean Meteor. Soc., 42, 129-151.

_- Y. Noh, and J. Dudhia, 2006: A new vertical diffusion package with an explicit treatment of entrainment processes. Mon. Wea. Rev., 134, 2318-2341, https://doi.org/10.1175/MWR3199.1.

Iacono, M. J., J. S. Deleware, E. J. Mlawer, M. W. Shepherd, S. A. Clough, and W. D. Collins, 2008: Radiative forcing by longlived greenhouse gases: Calculations with the AER radiative transfer models. J. Geophys. Res., 113, D13103, https://doi.org/ 10.1029/2008JD009944.

Judt, F., and S. S. Chen, 2016: Predictability and dynamics of tropical cyclone rapid intensification deduced from high-resolution stochastic ensembles. Mon. Wea. Rev., 144, 4395-4420, https:// doi.org/10.1175/MWR-D-15-0413.1.

, — - and J. Berner, 2016: Predictability of tropical cyclone intensity: Scale-dependent forecast error growth in high-resolution stochastic kinetic-energy backscatter ensembles. Quart. J. Roy. Meteor. Soc., 142, 43-57, https://doi.org/10.1002/qj.2626.

Kaplan, J., and M. DeMaria, 2003: Large-scale characteristics of rapidly intensifying tropical cyclones in the North Atlantic basin. Wea. Forecasting, 18, 1093-1108, https://doi.org/ 10.1175/1520-0434(2003)018<1093:LCORIT > 2.0.CO;2.

,-- , and J. A. Knaff, 2010: A revised tropical cyclone rapid intensification index for the Atlantic and eastern North Pacific basins. Wea. Forecasting, 25, 220-241, https://doi.org/10.1175/ 2009WAF2222280.1.

, and Coauthors, 2015: Evaluating environmental impacts on tropical cyclone rapid intensification predictability utilizing statistical models. Wea. Forecasting, 30, 1374-1396, https://doi.org/ 10.1175/WAF-D-15-0032.1.
Kimberlain, T. B., E. S. Blake, and J. P. Cangialosi, 2016: Tropical cyclone report: Hurricane Patricia (EP202015) 20-24 October 2015. National Hurricane Center Rep. EP202015, 32 pp., www.nhc.noaa.gov/data/tcr/EP202015_Patricia.pdf.

Lee, C.-Y., and S. S. Chen, 2012: Symmetric and asymmetric structures of hurricane boundary layer in coupled atmospherewave-ocean models and observations. J. Atmos. Sci., 69, 35763594, https://doi.org/10.1175/JAS-D-12-046.1.

Lin, I.-I., C.-H. Chen, I.-F. Pun, W. T. Liu, and C.-C. Wu, 2009: Warm ocean anomaly, air sea fluxes, and the rapid intensification of Tropical Cyclone Nargis. Geophys. Res. Lett., 36, L03817, https://doi.org/10.1029/2008GL035815.

Liu, Q., T. Marchok, H.-L. Pan, M. Bender, and S. Lord, 2000: Improvements in hurricane initialization and forecasting at NCEP with global and regional (GFDL) models. NOAA Tech. Procedures Bull. 472, 7 pp., http://www.nws.noaa.gov/ $\mathrm{om} / \mathrm{tpb} / 472 . p d f$.

Munsell, E. B., F. Zhang, J. A. Sippel, S. A. Braun, and Y. Weng, 2017: Dynamics and predictability of the intensification of Hurricane Edouard (2014). J. Atmos. Sci., 74, 573-595, https:// doi.org/10.1175/JAS-D-16-0018.1.

Rappaport, E. N., and Coauthors, 2009: Advances and challenges at the National Hurricane Center. Wea. Forecasting, 24, 395 419, https://doi.org/10.1175/2008WAF2222128.1.

Riemer, M., and M. T. Montgomery, 2011: Simple kinematic models for the environmental interaction of tropical cyclones in vertical wind shear. Atmos. Chem. Phys., 11, 9395-9414, https://doi.org/10.5194/acp-11-9395-2011.

Rodgers, E. B., W. S. Olson, V. M. Karyampudi, and H. F. Pierce, 1998: Satellite-derived latent heating distribution and environmental influences in Hurricane Opal (1995). Mon. Wea. Rev., 126, 1229-1247, https://doi.org/10.1175/1520-0493(1998)126<1229: SDLHDA $>2.0 . \mathrm{CO} ; 2$.

Rogers, R. F., 2010: Convective-scale structure and evolution during a high-resolution simulation of tropical cyclone rapid intensification. J. Atmos. Sci., 67, 44-70, https://doi.org/ 10.1175/2009JAS3122.1.

_ - P. D. Reasor, and J. A. Zhang, 2015: Multiscale structure and evolution of Hurricane Earl (2010) during rapid intensification. Mon. Wea. Rev., 143, 536-562, https://doi.org/ 10.1175/MWR-D-14-00175.1.

_- , and Coauthors, 2017: Rewriting the tropical record books: The extraordinary intensification of Hurricane Patricia (2015). Bull. Amer. Meteor. Soc., 98, 2091-2012, https://doi.org/ 10.1175/BAMS-D-16-0039.1.

Selz, T., and G. C. Craig, 2015: Upscale error growth in a highresolution simulation of a summertime weather event over Europe. Mon. Wea. Rev., 143, 813-827, https://doi.org/10.1175/ MWR-D-14-00140.1.

Shutts, G., 2005: A kinetic energy backscatter algorithm for use in ensemble prediction systems. Quart. J. Roy. Meteor. Soc., 131, 3079-3102, https://doi.org/10.1256/qj.04.106.

Skamarock, W. C., 2004: Evaluating mesoscale NWP models using kinetic energy backscatter. Mon. Wea. Rev., 132, 3019-3032, https://doi.org/10.1175/MWR2830.1.

- - , and Coauthors, 2008: A description of the Advanced Research WRF version 3. NCAR Tech. Note NCAR/ TN-475+STR, 113 pp., https://dx.doi.org/10.5065/D68S4MVH.

Steranka, J., E. B. Rodgers, and R. C. Gentry, 1986: The relationship between satellite-measured convective bursts and tropical cyclone intensification. Mon. Wea. Rev., 114, 1539-1546, https://doi.org/10.1175/1520-0493(1986)114<1539: TRBSMC $>2.0 . \mathrm{CO} ; 2$. 
Stevenson, S. N., K. L. Corbosiero, and J. Molinari, 2014: The convective evolution and rapid intensification of Hurricane Earl (2010). Mon. Wea. Rev., 142, 4364-4380, https://doi.org/ 10.1175/MWR-D-14-00078.1.

Tiedtke, M., 1989: A comprehensive mass flux scheme for cumulus parameterization in large-scale models. Mon. Wea. Rev., 117, 1779-1800, https://doi.org/10.1175/1520-0493(1989)117<1779: $\mathrm{ACMFSF}>2.0 . \mathrm{CO} ; 2$.

Zhang, C., Y. Wang, and K. Hamilton, 2011: Improved representation of boundary layer clouds over the southeast Pacific in ARQ-WRF using a modified Tiedtke cumulus parameterization scheme. Mon. Wea. Rev., 139, 3489-3513, https://doi.org/ 10.1175/MWR-D-10-05091.1.
Zhang, D.-L., and E. Altshuler, 1999: The effects of dissipative heating on hurricane intensity. Mon. Wea. Rev., 127, 30323038, https://doi.org/10.1175/1520-0493(1999)127<3032: TEODHO $>2.0 . \mathrm{CO} ; 2$.

Zhang, F., and J. A. Sippel, 2009: Effects of moist convection on hurricane predictability. J. Atmos. Sci., 66, 1944-1961, https:// doi.org/10.1175/2009JAS2824.1.

— dictability of tropical cyclones. J. Atmos. Sci., 70, 975-983, https://doi.org/10.1175/JAS-D-12-0133.1.

C. Snyder, and R. Rotunno, 2003: Effects of moist convection on mesoscale predictability. J. Atmos. Sci., 60, 1173-1185, https://doi.org/ 10.1175/1520-0469(2003)060<1173:EOMCOM>2.0.CO;2. 